\title{
LA FORMULE DE LA VARIATION SECONDE DE L'ENERGIE AU VOISINAGE D'UNE APPLICATION HARMONIQUE
}

\author{
E. MAZET
}

\section{Introduction}

Soit $(M, g)$ une variété riemannienne compacte connexe. Nous aurons à considérer sur $(M, g)$ des tenseurs du second ordre, soit symétriques, soit antisymétriques. Nous définissons des produits scalaires locaux de tels tenseurs par

$$
(h, k)=\frac{1}{2} h^{i j} k_{i j},
$$

ainsi que les produits scalaires globaux correspondants:

$$
\langle h, k\rangle=\int_{M}(h, k) .
$$

Si $\left(M^{\prime}, g^{\prime}\right)$ est une autre variété riemannienne, connexe et complète, considérons une application $C^{\infty}$, soit $f$, de $M$ dans $M^{\prime}$. Sur le fibré vectoriel $f^{*} T M^{\prime}$, de base $M$, nous pouvons mettre la structure riemannienne induite par $g^{\prime}$ (la fibre de $f^{*} T M^{\prime}$ en $x \in M$ n'étant autre que $\left.T_{f(x)} M^{\prime}\right)$. Nous avons encore des produits scalaires locaux et globaux pour les tenseurs sur $f^{*} T M^{\prime}$, définis par des formules identiques à (1) et $\left(1^{\prime}\right)$, mais à l'aide de la métrique $g^{\prime}$ et non plus de la métrique $g$. Ces produits scalaires étant définis sur des espaces différents, ce qui écarte toute ambiguité, nous les dénoterons par la même notation.

Cela étant, nous rappelons que l'énergie de $f$ est l'intégrale

$$
E(f)=\int_{M}\left(g, f^{*} g^{\prime}\right)=\left\langle g, f^{*} g^{\prime}\right\rangle .
$$

Dans $\S 1$, nous rappelons d'abord le calcul de la variation première de l'énergie, et la notion d'application harmonique. Puis nous calculons la variation seconde. Plus précisément, si $\mathscr{C}^{\infty}\left(M, M^{\prime}\right)$ désigne l'ensemble des applications $C^{\infty}$ de $M$ dans $M^{\prime}$, on sait que cet ensemble muni de la topologie $C^{\infty}$ peut être muni de façon naturelle d'une structure de variété fréchétique dont l'espace vectoriel tangent en $f$ est l'espace des sections $C^{\infty}$ de $f^{*} T M^{\prime}$. Cet

Communicated by A. Lichnerowicz, January 7, 1972. 
espace vectoriel sera noté $\Gamma\left(f^{*} T M^{\prime}\right)$. Soient $u$ et $v$ deux éléments de $\Gamma\left(f^{*} T M^{\prime}\right)$. On appellera variation de $f$ issue de $(u, v)$ toute application $\lambda, C^{\infty}$, de $M \times$ ]$-\varepsilon, \varepsilon[\times]-\varepsilon, \varepsilon\left[\right.$, avec $\varepsilon>0$, dans $M^{\prime}$, notée $(x, s, t) \rightarrow \lambda(x ; s, t)$, telle que

$$
\lambda(x ; 0,0)=f(x), \quad \frac{\partial \lambda}{\partial s}(x ; 0,0)=u_{x}, \quad \frac{\partial \lambda}{\partial t}(x ; 0,0)=v_{x}
$$

pour tout $x \in M$. Alors, appelant $E(s, t)$ l'énergie de l'application $x \rightarrow \lambda(x ; s, t)$, on a en supposant que $f$ est harmonique (i.e., un point critique de l'énergie)

$$
\left.\frac{\partial^{2} E(s, t)}{\partial s \partial t}\right|_{s=t=0}=\langle u, L v\rangle,
$$

où $L: \Gamma\left(f^{*} T M^{\prime}\right) \rightarrow \Gamma\left(f^{*} T M^{\prime}\right)$ est un opérateur différentiel linéaire d'ordre 2 , elliptique, autoadjoint.

On démontre que $L$ admet un indice au sens des formes quadratiques, c'est-à-dire qu'il existe dans $\Gamma\left(f^{*} T M^{\prime}\right)$ un sous-espace de codimension finie sur lequel la forme quadratique $u \rightarrow\langle u, L u\rangle$ est définie positive (l'indice est alors défini comme le maximum des dimensions des sous-espaces sur lesquels la dite forme est définie négative: c'est un entier $\geq 0$ ).

Lorsque $\left(M^{\prime}, g^{\prime}\right)$ est à courbure sectionnelle non positive au voisinage de $f(M), f$ étant harmonique, on décrit le comportement de l'énergie au voisinage de $f$, et on caractérise simplement les applications harmoniques voisines. On obtient ainsi une version locale de résultats qui n'étaient connus que sous des hypothèses globales. On obtient des renseignements sur le rang d'une application harmonique, et sur le type d'homotopie de $\mathscr{C}^{\infty}\left(M, M^{\prime}\right)$.

Dans la $\S 2$, nous donnons les résultats homologues dans le cas complexe.

Dans $\S 3$, on se place dans le cas particulier où $\left(M^{\prime}, g^{\prime}\right)=(M, g)$ et où $f$ est l'identité. On peut alors préciser le théorème classique de Bochner sur les isométries des variétés compactes à courbure négative par un résultat de rigidité. On obtient également une caractérisation purement variationnelle des isométries infinitésimales.

\section{L'operateur de la variation seconde}

Commencons par calculer la variation première de l'énergie. Soit $u \epsilon$ $\Gamma\left(f^{*} T M^{\prime}\right)$, et considérons une variation $\lambda(x ; t)$ issue de $u$. On a

$$
E(t)=\int_{M}\left(g, \lambda_{t}^{*} g^{\prime}\right)
$$

où

$$
\lambda_{t}(x)=\lambda(x ; t) .
$$


Choisissons un recouvrement de $M$ par des ouverts $\left\{\mathfrak{U}_{\alpha}\right\}_{\alpha \in A}$ tels que sur chaque $\mathfrak{U}_{\alpha}$ on puisse choisir un champ $C^{\infty}$ de bases orthonormées de $(M, g)$, soit $\left\{X_{1}^{\alpha}, \cdots, X_{n}^{\alpha}\right\}$. Alors, dans $\mathfrak{U}_{\alpha}$, pour tout choix des $X_{i}^{\alpha}$ on a

$$
\left(g, \lambda_{t}^{*} g^{\prime}\right)=\frac{1}{2} \sum_{i=1}^{n}\left(\lambda_{t^{*}} X_{i}^{\alpha}, \lambda_{t^{*}} X_{i}^{\alpha}\right)
$$

Nous écrirons désormais, pour ne pas alourdir les notations

$$
E(t)=\frac{1}{2} \int_{M}\left(\lambda_{*} X_{i}, \lambda_{*} X_{i}\right)
$$

étant entendu que $\lambda_{*}=\lambda_{t^{*}}$, et qu'il $y$ a sommation de $i=1$ à $n$.

Désignons par $D$ et $D^{\prime}$ respectivement les connexions canoniques de $(M, g)$ et $\left(M^{\prime}, g^{\prime}\right)$. On a alors

$$
\frac{d E}{d t}=\frac{1}{2} \int_{M} \frac{\partial}{\partial t}\left(\lambda_{*} X_{i}, \lambda_{*} X_{i}\right)=\int_{M}\left(\frac{D^{\prime}}{\partial t} \lambda_{*} X_{i}, \lambda_{*} X_{i}\right)=\int_{M}\left(D^{\prime}{ }_{X_{i}} \frac{\partial \lambda}{\partial t}, \lambda_{*} X_{i}\right),
$$

puisque $\left[\partial / \partial t, X_{i}\right]=0$ sur $\left.M \times\right]-\varepsilon, \varepsilon[$. Puis

$$
\frac{d E}{d t}=\int_{M}\left[X_{i} \cdot\left(\frac{\partial \lambda}{\partial t}, \lambda_{*} X_{i}\right)-\left(\frac{\partial \lambda}{\partial t}, D_{X_{i}}^{\prime} \lambda_{*} X_{i}\right)\right] .
$$

Définissons une 1-forme sur $M$ par $\omega_{t}(X)=\left(\partial \lambda / \partial t, \lambda_{*} X\right)$. Soit $\omega_{t}^{\sharp}$ le vecteur image de $\omega_{t}$ par l'isomorphisme de $T^{*} M$ sur $T M$ associé à $g$. Alors, $X_{i} \cdot\left(\partial \lambda / \partial t, \lambda_{*} X_{i}\right)=X_{i} \cdot\left(\omega_{t}^{\#}, X_{i}\right)=\left(D_{X_{i}} \omega_{t}^{\sharp}, X_{i}\right)+\left(\omega_{t}^{\sharp}, D_{X_{i}} X_{i}\right)$. Dans cette somme, le premier terme est une divergence, qui disparaît par intégration. Le second terme vaut $\left(\partial \lambda / \partial t, \lambda_{*} D_{X_{i}} X_{i}\right)$.

Il reste sous le signe d'intégration le produit scalaire de $\partial \lambda / \partial t$ par un vecteur qui s'écrit $\lambda_{*} \sum_{i=1}^{n} D_{X_{i}} X_{i}-\sum_{i=1}^{n} D_{X_{i}}^{\prime} \lambda_{*} X_{i}$, dont on vérifiera aisément qu'il ne dépend pas du choix des $X_{i}$. On notera ce vecteur, pour $t=0$, par $-\tau(f)$. Rappelons que c'est un élément de $\Gamma\left(f^{*} T M^{\prime}\right)$. On aura finalement

$$
\left.\frac{d E}{d t}\right|_{t=0}=-\int_{M}(u, \tau(f))=-\langle u, \tau(f)\rangle \text {. }
$$

avec

$$
\tau(f)=\sum_{i=1}^{n} D_{X_{i}}^{\prime} f_{*} X_{i}-f_{*}\left(\sum_{i=1}^{n} D_{X_{i}} X_{i}\right)
$$

Ce $\tau(f)$ est le "champ de tension" selon la terminologie de [4].

Les applications $f$ qui sont points critiques de l'énergie sont donc celles qui vérifient l'équation 


$$
\tau(f)=0
$$

et on sait qu'on leur donne le non d'applications harmoniques.

Soient maintenant $u, v \in \Gamma\left(f^{*} T M^{\prime}\right)$ et soit $\lambda(x ; s, t)$ une variation issue de $(u, v)$. Soit à calculer la variation seconde $\left.\frac{\partial^{2} E(s, t)}{\partial s \partial t}\right|_{s=t=0}$. Repartons de la relation $\frac{\partial E}{\partial t}=\int_{M}\left(D^{\prime}{ }_{X_{i}} \frac{\partial \lambda}{\partial t}, \lambda_{*} X_{i}\right)$. Alors

$$
\begin{aligned}
\frac{\partial^{2} E}{\partial s \partial t}=\int_{M} \frac{\partial}{\partial s}\left(D^{\prime}{ }_{X_{i}} \frac{\partial \lambda}{\partial t}, \lambda_{*} X_{i}\right)=\int_{M} & {\left[\left(\frac{D^{\prime}}{\partial s} D^{\prime}{ }_{X_{i}} \frac{\partial \lambda}{\partial t}, \lambda_{*} X_{i}\right)\right.} \\
+ & \left.\left(D^{\prime}{ }_{X_{i}} \frac{\partial \lambda}{\partial t}, \frac{D^{\prime}}{\partial s} \lambda_{*} X_{i}\right)\right] .
\end{aligned}
$$

On a (cf. [1, théorème II.5.12])

$$
\frac{D^{\prime}}{\partial s} D^{\prime}{ }_{X_{i}} \frac{\partial \lambda}{\partial t}=D_{X_{i}}^{\prime} \frac{D^{\prime}}{\partial s} \frac{\partial \lambda}{\partial t}-R^{\prime}\left(\frac{\partial \lambda}{\partial s}, \lambda_{*} X_{i}\right) \frac{\partial \lambda}{\partial t},
$$

où $R^{\prime}$ est l'opérateur de courbure sur $\left(M^{\prime}, g^{\prime}\right)$. Alors

$$
\left(\frac{D^{\prime}}{\partial s} D^{\prime}{ }_{X_{i}} \frac{\partial \lambda}{\partial t}, \lambda_{*} X_{i}\right)=\left(D_{X_{i}}^{\prime} \frac{D^{\prime}}{\partial s} \frac{\partial \lambda}{\partial t}, \lambda_{*} X_{i}\right)-R^{\prime}\left(\frac{\partial \lambda}{\partial s}, \lambda_{*} X_{i}, \frac{\partial \lambda}{\partial t}, \lambda_{*} X_{i}\right) .
$$

Le premier termes'écrit

$$
\begin{aligned}
& X_{i}\left(\frac{D^{\prime}}{\partial s} \frac{\partial \lambda}{\partial t}, \lambda_{*} X_{i}\right)-\left(\frac{D^{\prime}}{\partial s} \frac{\partial \lambda}{\partial t}, D^{\prime}{ }_{X_{i}} \lambda_{*} X_{i}\right) \\
&=\operatorname{div} \bar{\omega}_{s, t}^{\sharp}+\left(\frac{D^{\prime}}{\partial s} \frac{\partial \lambda}{\partial t}, \lambda_{*} D_{X_{i}} X_{i}-D_{X_{i} \lambda_{*}}^{\prime} X_{i}\right) \\
&:=\operatorname{div} \bar{\omega}_{s, t}^{\sharp}-\left(\frac{D^{\prime}}{\partial s} \frac{\partial \lambda}{\partial t}, \tau\left(\lambda_{s}, t\right)\right),
\end{aligned}
$$

où l'on a défini la 1 -forme $\bar{\omega}_{s, t}$ sur $M$ par $\bar{\omega}_{s, t}(X)=\left(\frac{D^{\prime}}{\partial s} \frac{\partial \lambda}{\partial t}, \lambda_{*} X\right)$. Par ailleurs,

$$
\begin{aligned}
\left(D^{\prime}{ }_{X i} \frac{\partial \lambda}{\partial t}, \frac{D^{\prime}}{\partial s} \lambda_{*} X_{i}\right) & =\left(D^{\prime}{ }_{X_{i}} \frac{\partial \lambda}{\partial t}, D_{X_{i}}^{\prime} \frac{\partial \lambda}{\partial s}\right) \\
& =X_{i} \cdot\left(\frac{\partial \lambda}{\partial s}, D_{X_{i}}^{\prime} \frac{\partial \lambda}{\partial t}\right)-\left(\frac{\partial \lambda}{\partial s}, D_{X_{i}}^{\prime} D_{X_{i}}^{\prime} \frac{\partial \lambda}{\partial t}\right) \\
& =\operatorname{div} \alpha_{s, t}^{*}+\left(\frac{\partial \lambda}{\partial s}, D_{D_{X_{i}} X_{i}}^{\prime} \frac{\partial \lambda}{\partial s}\right)-\left(\frac{\partial \lambda}{\partial s}, D_{X_{i}}^{\prime} D_{X_{i}}^{\prime} \frac{\partial \lambda}{\partial t}\right)
\end{aligned}
$$


où l'on a défini la 1 -forme $\alpha_{s, t} \operatorname{sur} M \operatorname{par} \alpha_{s, t}(X)=\left(\partial \lambda / \partial s, D_{X}^{\prime}(\partial x / \partial t)\right)$. Il vient enfin

$$
\frac{\partial^{2} E}{\partial s \partial t}=\int_{M}\left[-\left(\frac{D^{\prime}}{\partial s} \frac{\partial \lambda}{\partial t}, \tau\left(\lambda_{s, t}\right)\right)+\left(\frac{\partial \lambda}{\partial s}, L_{s, t} \frac{\partial \lambda}{\partial t}\right)\right]
$$

où

$$
\begin{aligned}
L_{s, t} \frac{\partial \lambda}{\partial t}= & D_{\sum_{i=1}^{\prime} D_{X_{i} X_{i}}} \frac{\partial \lambda}{\partial t}-\sum_{i=1}^{n} D_{X_{i}}^{\prime} D_{X_{i}}^{\prime} \frac{\partial \lambda}{\partial t} \\
& -\sum_{i=1}^{n} R^{\prime}\left(\lambda_{s, t^{*}} X_{i}, \frac{\partial \lambda}{\partial t}\right) \lambda_{s, t^{*}} X_{i}
\end{aligned}
$$

Ici encore, on vérifie aisément que l'opérateur $L_{s, t}$ ne dépend pas du choix $\operatorname{des} X_{i}$. En particulier, pour $s=t=0$, et $f$ harmonique,

$$
\left.\frac{\partial^{2} E}{\partial s \partial t}\right|_{s=t=0}=\int_{M}(u, L v)=\langle u, L v\rangle
$$

avec

$$
L v=D_{\sum_{i=1}^{n} D_{X_{i}} X_{i}}^{\prime} v-\sum_{i=1}^{n} D_{X_{i}}^{\prime} D_{X_{i}}^{\prime} v-\sum_{i=1}^{n} R^{\prime}\left(f_{*} X_{i}, v\right) f_{*} X_{i}
$$

Du calcul précédent résulte en ontre la formule

$$
\begin{aligned}
\frac{\partial^{2} E}{\partial s \partial t}= & -\int_{M}\left(\frac{D^{\prime}}{\partial s} \frac{\partial \lambda}{\partial t}, \tau\left(\lambda_{s t}\right)\right)+\int_{M} \sum_{i=1}^{n}\left(D_{X_{i}}^{\prime} \frac{\partial \lambda}{\partial t}, D_{X_{i}}^{\prime} \frac{\partial \lambda}{\partial t}\right) \\
& -\int_{M} \sum_{i=1}^{n} R^{\prime}\left(\lambda_{*} X_{i}, \frac{\partial \lambda}{\partial s}, \lambda_{*} X_{i}, \frac{\partial \lambda}{\partial t}\right) .
\end{aligned}
$$

Un calcul en coordonnées locales, fastidieux mais standard montre que $L$ est un opérateur différentiel linéaire elliptique du second ordre. Il est autoadjoint car

$$
\left.\frac{\partial^{2} E}{\partial s \partial t}\right|_{s=t=0}=\left.\frac{\partial^{2} E}{\partial t \partial s}\right|_{s=t=0} .
$$

Proposition 1. La forme quadratique $u \rightarrow\langle u, L u\rangle$ sur $\Gamma\left(f^{*} T M^{\prime}\right)$ admet un indice.

Observons que, puisque $L$ est elliptique autoadjoint, la forme quadratique considérée admet un indice si et seulement si les valeurs propres de $L$ sont bornées inférieurement. La démonstration s'inspire alors de [7, pp. 134-135].

Définissons une fonction continue $\theta$ sur $M$ comme suit: pour $x \in M$, soit 
$Q_{x}$ l'endomorphisme autoadjoint de $T_{f(x)} M^{\prime}$ défini par

$$
u \rightarrow-\sum_{i=1}^{n} R^{\prime}\left(f_{*} X_{i}, u\right) f_{*} X_{i} .
$$

Alors, on prend pour $\theta(x)$ la plus petite valeur propre de $Q_{x}$ (ces valeurs propres étant réelles),

Soit $\beta$ le minimum de $\theta$ sur le compact $M$. Supposons que $L$ admette une valeur propre $\alpha<\beta$, et soit $u \neq 0$ un vecteur propre correspondant. D'après la formule (7), on a

$$
\langle u, \alpha u-Q u\rangle=\langle u, L u-Q u\rangle=\int_{M} \sum_{i=1}^{n}\left(D^{\prime}{ }_{X_{i}} u, D^{\prime}{ }_{X_{i}} u\right) \geq 0 .
$$

Soit $\alpha\langle u, u\rangle \geq\langle u, Q u\rangle$. Or, de par le choix de $\alpha$, on a $\alpha(u, u) \leq(u, Q u)$, avec l'inégalité stricte partout où $u$ n'est pas nul. Donc $\alpha\langle u, u\rangle\langle\langle u, Q u\rangle$, d'où contradiction. Ainsi, toute valeur propre de $L$ est au moins égale à $\beta$.

Etudions maintenant le cas où $\left(M^{\prime}, g^{\prime}\right)$ est à courbure sectionnelle non positive au voisinage de $f(M)$. Auparavant, donnons une définition.

Définition 1. L'application $f:(M, g) \rightarrow\left(M^{\prime}, g^{\prime}\right)$ sera dite localement statistiquement incontractable (en abrégé LSIC) s'il existe un voisinage $\mathfrak{A}$ de $f$ dans $\mathscr{C}^{\infty}\left(M, M^{\prime}\right)$ tel que pour toute $h \in \mathfrak{A}$, on ait $E(h) \geq E(f)$. Elle sera dite globalement statistiquement incontractable (GSIC) si pour toute $h \in \mathscr{C}^{\infty}\left(M, M^{\prime}\right)$, $h$ homotope à $f$, on a $E(h) \geq E(f)$.

Notes. 1) La définition de LSIC suppose le choix d'une topologie sur $\mathscr{C}^{\infty}\left(M, M^{\prime}\right)$, et cet espace est suceptible de recevoir diverses topologies: topologies $C^{k}$, avec $k=0,1, \cdots, \infty$. Nous dirons donc que $f$ est LSIC au sens $C^{k}$ si le voisinage $\mathfrak{A}$ dont il est question est un voisinage pour la topologie $C^{k}$.

2) La terminologie "LSIC", "GSIC", se réfère à l'interprétation de l'énergie donnée en [4, p. 114]. Il est clair que toute application LSIC est harmonique. Il est aisé de voir que toute application GSIC est aussi LSIC, pour toute topologie sur $\mathscr{C}^{\infty}\left(M, M^{\prime}\right)$.

Proposition 2. Soit $f:(M, g) \rightarrow\left(M^{\prime}, g^{\prime}\right)$ une application harmonique.

a) $S i\left(M^{\prime}, g^{\prime}\right)$ est à courbure sectionnelle non positive sur $f(M)$, l'opérateur $L$ est semi-défini positif, et son noyau est formé des champs parallèles le long de $f$. En particulier si $\xi$ est une isométrie infinitésimale de $(M, g), f_{*} \xi$ est parallèle le long de $f$, et si $\xi^{\prime}$ est une isométrie infinitésimale de $\left(M^{\prime}, g^{\prime}\right), \xi^{\prime} \circ f$ est parallèle le long de $f$.

b) Si $\left(M^{\prime}, g^{\prime}\right)$ est à courbure sectionnelle non positive au voisinage de $f(M)$, $f$ est LSIC au sens $C^{0}$. Il existe même dans $\mathscr{C}^{\infty}\left(M, M^{\prime}\right)$ un voisinage $\mathfrak{A}$ de $f$ au sens $C^{0}$ tel que l'on ait $E(h) \geq E(f)$ pour toute $h \in \mathfrak{A}$, l'égalité étant atteinte si et seulement si hest harmonique, auquel cas il existe un champ $u \in \Gamma\left(f^{*} T M^{\prime}\right)$, parallèle le long de $f$, tel que l'on ait $h=\exp _{f}^{\prime} u$; et de plus les applications 
intermédiaires $\exp _{f}^{\prime} s u, s \in[0,1]$, sont harmoniques.

c) Si $\left(M^{\prime}, g^{\prime}\right)$ est à courbure sectionnelle strictement négative sur $f(M), f$ est isolée dans l'ensemble des applications harmoniques pour la topologie $C^{0}$, sauf peut être si $f$ est de rang $\leq 1$. Si en outre le premier nombre de Betti $b_{1}(M)$ est nul, $f$ est isolée dans l'ensemble des applications harmoniques, sauf si elle est constante. (Par $\exp _{f}^{\prime} u$, nous entendons l'application $x \rightarrow \exp _{f(x)}^{\prime} u_{x}$. Nous disons qu'un champ $u \in \Gamma\left(f^{*} T M^{\prime}\right)$ est parallèle le long de $f$ si $D_{X}^{\prime} u=0$ pour tout vecteur $X \in T M$ ).

a) D'après la formule (7), il est clair que pour tout $u \in \Gamma\left(f^{*} T M^{\prime}\right),\langle u, L u\rangle$ $\geq 0$. Alors $L u=0$ équivaut à $\langle u, L u\rangle=0$, et cela entraîne $\int_{M} \sum_{i=1}^{n}\left(D^{\prime}{ }_{X_{i}} u, D^{\prime}{ }_{X_{i}} u\right)=o$, d'où $D_{X_{i}}^{\prime} u=o$ pour tout choix des $X_{i}$. Donc $u$ est paralléle le long de $f$. Par ailleurs, posons

$$
\lambda(x ; s)=\left\{\begin{array}{l}
f\left(\exp _{x} s \xi_{x}\right) \text { si } \xi \text { est une isométrie infinitésimale de }(M, g), \\
\exp _{f(x)} s \xi_{f(x)}^{\prime} \text { si } \xi^{\prime} \text { est une isométrie infinitésimale de }\left(M^{\prime}, g^{\prime}\right) .
\end{array}\right.
$$

Dans les deux cas on a $E(s)=$ cte, donc en prenant la dérivée seconde

$$
\left\langle f_{*} \xi, L f_{*} \xi\right\rangle=0 \quad \text { et } \quad\left\langle\xi^{\prime} \circ f, L \xi^{\prime} \circ f\right\rangle=0,
$$

donc $f_{*} \xi$ et $\xi^{\prime} \circ f$ sont parallèles le long de $f$.

b) Choisissons $\varepsilon>0$ tel que pour tout $x \in M$, la boule de centre $f(x)$ et de rayon $\varepsilon$ dans $\left(M^{\prime}, g^{\prime}\right)$ soit un voisinage normal de $f(x)$. Un tel $\varepsilon$ existe car $f(M)$ est compact. On peut en outre supposer $\varepsilon$ assez petit pour que les boules en question soient toutes dans un voisinage de $f(M)$ sur lequel la courbure sectionnelle est non positive.

Soit alors $\mathfrak{U}$ la boule de centre $f$ et de rayon $\varepsilon$ dans $\mathscr{C}^{\infty}\left(M, M^{\prime}\right)$ muni de la métrique de la convergence uniforme. Soit $h \in \mathfrak{A}$. Pour tout $x \in M$ il existe un arc géodésique minimisant de $x$ à $f(x)$. Notons le $s \rightarrow \lambda(x ; s)$. On a d'après la formule (5);

$$
\frac{d^{2} E}{d s^{2}}=\int_{M}\left[-\left(\frac{D^{\prime}}{\partial s} \frac{\partial \lambda}{\partial s}, \tau\left(\lambda_{s}\right)\right)+\left(\frac{\partial \lambda}{\partial s}, L_{s} \frac{\partial \lambda}{\partial s}\right)\right]
$$

Par construction $\left(D^{\prime} / \partial s\right)(\partial \lambda / \partial s)=0$. Il reste

$$
\frac{d^{2} E}{d s^{2}}=\int_{M} \sum_{i=1}^{n}\left(D^{\prime}{ }_{X_{i}} \frac{\partial \lambda}{\partial s}, D_{X_{i}}^{\prime} \frac{\partial \lambda}{\partial s}\right)-\int_{M} \sum_{i=1}^{n} R^{\prime}\left(\lambda_{s^{*}} X_{i}, \frac{\partial \lambda}{\partial s}, \lambda_{s^{*}} X_{i}, \frac{\partial \lambda}{\partial s}\right) \geq 0 .
$$

Comme de plus $d E /\left.d s\right|_{s=0}=0$, on a $d E / d s \geq 0$ pour tout $s$. Donc $E(h) \geq E(f)$, et $f$ est LSIC au sens $C^{0}$. 
Si $E(h)=E(f)$, alors $h$ est aussi LSIC, donc harmonique. En outre $E(s)$ est constant, et chacune des $\lambda_{s}$ est harmonique. Posant $u_{x}=(\partial \lambda / \partial s)(x ; 0)$, on a $\langle u, L u\rangle=0$, donc $u$ est parallèle le long de $f$, et l'on a $h=\exp _{f}^{\prime} u$.

c) Il ressort de la démonstration de b) que, sous les hypothèses faites, $f$ est isolée si et seulement si $L$ est défini positif. Supposons donc que $L$ ne soit pas défini positif, c'est-à-dire que son noyau contienne un élément $u \neq 0$. Comme $u$ est parallèle le long de $f$, et que $M$ est connexe, $u$ est de norme constante, que l'on peut supposer égale à 1 . On a pour toute base $\left\{X_{i}\right\}_{i=1}^{n}$ au point $x \in M$,

$$
R^{\prime}\left(f_{*} X_{i}, u, f_{*} X_{i}, u\right)=0
$$

donc $f_{*} X_{i}$ est colinéaire à $u$. Alors $f$ est partout de rang au plus égal à 1 . Pour tout $x \in M$, il existe une forme linéaire $\omega_{x}$ sur $T_{x} M$ telle que l'on ait

$$
f_{*} X=\omega_{x}(X) \cdot u_{x} \quad \text { pour tout } X \in T_{x} M .
$$

La collection de $\omega_{x}$ définit une 1-forme sur $M$. On a encore $\omega(X)=\left(f_{*} X, u\right)$. Pour deux champs $X, Y$ sur $M$, on a

$$
\begin{aligned}
d \omega(X, Y) & =X \cdot \omega(Y)-Y \cdot \omega(X)-\omega([X, Y]) \\
& =\left(D^{\prime}{ }_{X} f_{*} Y-D^{\prime}{ }_{Y} f_{*} X-f_{*}[X, Y], u\right)=0 .
\end{aligned}
$$

Donc, $\omega$ est fermée.

Ecrivons d'autre part la nullité de $\tau(f)$, en choisissant une base orthonormée $\left\{X_{i}\right\}$ en un point $x$ de $M$, et en la prolongeant localement par parallélisme le long des géodésiques issues de $x$, de sorte que $D_{X_{i}} X_{i}=0$. Il vient $\sum_{i=1}^{n} D_{X_{i}}^{\prime} f_{*} X_{i}$ $=0, d^{\prime}$ où

$$
0=\sum_{i=1}^{n}\left(D_{X_{i}}^{\prime} f_{*} X_{i}, u_{x}\right)=\sum_{i=1}^{n} X_{i} \cdot\left(f_{*} X_{i}, u_{x}\right)=\sum_{i=1}^{n} X_{i} \cdot \omega\left(X_{i}\right)=\sum_{i=1}^{n} X_{i} \cdot\left(\omega^{\#}, X_{i}\right)
$$

soit finalement $0=\sum_{i}\left(D_{X_{i}} \omega^{\sharp}, X_{i}\right)=-\delta \omega$.

Donc $\omega$ est aussi cofermée. Finalement, $\omega$ est harmonique. Si $b_{1}(M)=0$, donc $\omega=0, f_{*}$ est partout nulle, et $f$ est constante.

Remarque 2.1. La partie a) donne une version locale de résultats purement globaux dus à Eells-Sampson [4] et Hartman [5] dans le cas où $\left(M^{\prime}, g^{\prime}\right)$ est à courbure sectionnelle non positive et complète. Dans ce cas toute application harmonique est GSIC. Si deux applications harmoniques sont homotopes, il existe une homotopie $(x, s) \rightarrow \lambda(x ; s)$ de l'une à l'autre telle que toutes les courbes $\lambda_{x}: s \rightarrow \lambda(x ; s)$ soit des géodésiques, et que toutes les applications $\lambda_{s}: x \rightarrow \lambda(x ; s)$ soient harmoniques.

Si $M$ est à premier nombre de Betti nul, et si $M^{\prime}$ est compacte et à courbure sectionnelle strictement négative, nous pouvons préciser le type d'homotopie de $\mathscr{C}^{\infty}\left(M, M^{\prime}\right)$. La composante connexe des applications constantes a le type 
d'homotopie de $M^{\prime}$. Les autres sont contractiles. Dans chaque cas, une équivalence d'homotopie est fournie par l'équation de la chaleur.

Remarque 2.2. On peut se demander si les deux types de champs envisagés à la partie a) engendrent le noyau de $L$. Il n'en est rien. Par exemple, prenons pour $(M, g)$ le cercle $\left(S^{1}, g_{0}\right)$ et pour $\left(M^{\prime}, g^{\prime}\right)$ la bandle de Mœbius, $\left(B, g_{0}\right)$, quotient de $\left(\boldsymbol{R}^{2}, g_{0}\right)$ par l'isométrie $(x, y) \rightarrow\left(x+\frac{1}{2},-y\right)$. Considérons l'application harmonique $f:\left(S^{1}, g_{0}\right) \rightarrow\left(B, g_{0}\right)$ définie par $f(s)=(2 s, 0)$.

Alors, les deux types de champs envisagés engendrent le même sous-espace, formés des champs proportionnels à $f_{*}(\partial / \partial s)$. Mais considérons le champ défini par $u_{s}=(0,1)$.

Il est clair que $u$ est dans Ker $L$, mais n'est pas colinéaire à $f_{*}(\partial / \partial s)$.

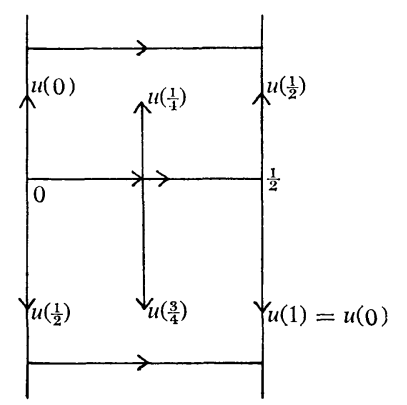

Remarque 2.3. On peut se demander, dans le cas où $\left(M^{\prime}, g^{\prime}\right)$ est à courbure sectionnelle non positive au voisinage de $f(M)$, si le noyau de $L$ est exactement l'ensemble des champs parallèles le long de $f$. On vérifie aisément qu'il en est ainsi lorsque $\left(M^{\prime}, g^{\prime}\right)$ est plate au voisinage de $f(M)$. Mais prenons pour $f$ une géodésique fermée d'une variété à courbure strictement négative de dimension paire orientable. Alors tout champ contenu dans le noyau de $L$ doit être tangent à $f$. Or, le transport parallèle le long de $f$ induit une rotation de l'orthogonal de $f(0)$ dans $T_{f(0)} M$, lequel est de dimension impaire. Cette rotation laisse un vecteur non nul fixe, et on en déduit l'existence d'un champ parallèle non nul orthogonal à $f$, lequel n'est pas dans $\operatorname{Ker} \boldsymbol{L}$.

Remarque 2.4. Les résultats de c) sont à mettre en parallèle avec certains résultats de Lichérowicz $[9$, p. 7$]$ : sous nos hypothèses, et si de plus la courbure de Ricci de $(M, g)$ est non négative, toute application harmonique est totalement géodésique; dans le cas correspondant à $\mathrm{c}$ ), toute application harmonique est constante, ou de rang constant 1 . N'ayant plus de condition sur la courbure de Ricci, nous devons nous attendre à ne pas avoir des résultats aussi forts. Montrons le par un contre exemple.

Soit $(M, g)$ une variètè riemannienne compacte connexe, et $\bar{\omega}$ une 1 -forme harmonique non nulle sur $(M, g)$. Nous supposons de plus que la classe de cohomologie de $\bar{\omega}$ dans $H^{1}(M, R)$ est rationnelle. Suivant [4, p. 128], on peut 
construire à l'aide de $\bar{\omega}$ une application harmonique de $(M, g)$ dans $\left(S^{1}, g_{0}\right): \bar{\omega}$ coincidera, à un facteur près, avec la forme $\omega$ qui intervient dans la démonstration du c) de la proposition 2.

Soit $\left(M^{\prime}, g^{\prime}\right)$ une variété riemannienne compacte queclonque. On peut toujours trouver une géodésique fermée $\left(S^{1}, g_{0}\right) \rightarrow\left(M^{\prime}, g^{\prime}\right)$. La composée des deux applications est une application harmonique de $(M, g)$ dans $\left(M^{\prime}, g^{\prime}\right)$, non constante, de rang au plus égal à 1 . La forme $\omega$ de proposition $2, c)$, proportionnelle à $\omega$, devra s'annuler si la caractéristique d'Euler-Poincaré de $(M, g)$ est non nulle.

On peut conclure que si $(M, g)$ est à premier nombre de Betti et à caractéristique d'Euler-Poincaré différents de 0 , alors, pour toute variété compacte $\left(M^{\prime}, g^{\prime}\right)$ il existe une application harmonique de $(M, g)$ dans $\left(M^{\prime}, g^{\prime}\right)$, non constante, de rang au plus égal à 1 , de rang non constant, et en particulier non totalement géodésique.

\section{Etude du cas Kählerien}

Ici $(M, g, J)$ et $\left(M^{\prime}, g^{\prime}, J^{\prime}\right)$ seront des variétés kählériennes ( $J$ et $J^{\prime}$ désignant les opérateurs de structure complexe: $J^{2}=J^{\prime 2}=-$ Id). Alors toute application holomorphe ou antiholomorphe de $(M, J)$ dans $\left(M^{\prime}, J^{\prime}\right)$ est une application harmonique de $(M, g)$ dans $\left(M^{\prime}, g^{\prime}\right)$.

Soit $f \in \mathscr{C}^{\infty}\left(M, M^{\prime}\right)$. Si $n$ désigne la dimension complexe de $M$, soit $\left\{X_{j}\right\}_{i=1}^{n}$ un champ local $\mathscr{C}^{\infty}$ de bases orthonormées complexes de $(M, g, J)$. On lui associe les vecteurs

$$
\begin{array}{r}
Q_{j}=\frac{1}{\sqrt{2}}\left(f_{*} X_{j}-J^{\prime} f_{*} J X_{j}\right), \quad \tilde{Q}_{j}=\frac{1}{\sqrt{2}}\left(f_{*} X_{j}+J^{\prime} f_{*} J X_{j}\right), \\
j=1, \cdots, n .
\end{array}
$$

Un calcul sans difficulté montre que les quantités $\sum_{j=1}^{n}\left(Q_{j}, Q_{j}\right)$ et $\sum_{j=1}^{n}\left(\tilde{Q}_{j}, \tilde{Q}_{j}\right)$ ne dépendent pas du choix de la base orthonormée complexe $\left\{X_{j}\right\}$. On définit alors les énergies partielles

$$
E^{\prime}(f)=\frac{1}{2} \int_{M} \sum_{j=1}^{n}\left(Q_{j}, Q_{j}\right), \quad E^{\prime \prime}(f)=\frac{1}{2} \int_{M}\left(\tilde{Q}_{j}, \tilde{Q}_{j}\right) .
$$

On vérifie trivialement que $f$ est holomorphe (resp. antiholomorphe) si seulement si les $\tilde{Q}_{j}$ (resp les $Q_{j}$ ) sont nuls, ou encore si $E^{\prime \prime}(f)$ (resp. $E^{\prime}(f)$ ) est nulle. On a d'autre part $E^{\prime}(f)+E^{\prime \prime}(f)=E(f)$ tandis que

$$
E^{\prime}(f)-E^{\prime \prime}(f)=-\int_{M} \sum_{j=1}^{n}\left(f_{*} X_{j}, J^{\prime} f_{*} J X_{j}\right)=\int_{M} \sum_{j=1}^{n}\left(J^{\prime} f_{*} X_{j}, f_{*} J X_{j}\right) .
$$

Dans les calculs qui suivent nous supprimerons $\sum_{j=1}^{n}$ par abus de notation. On a, le long d'une variation $(x, s) \rightarrow \lambda(x ; s)$, 


$$
\begin{aligned}
\frac{d E^{\prime}}{d s}-\frac{d E^{\prime \prime}}{d s} & =\int_{M} \frac{\partial}{\partial s}\left(J^{\prime} \lambda_{*} X_{j}, \lambda_{*} J X_{j}\right) \\
& =\int_{M}\left[\left(\frac{D^{\prime}}{\partial s} J^{\prime} \lambda_{*} X_{j}, \lambda_{*} J X_{j}\right)+\left(J^{\prime} \lambda_{*} X_{j}, \frac{D^{\prime}}{\partial s} \lambda_{*} J X_{j}\right)\right] .
\end{aligned}
$$

La variété $\left(M^{\prime}, g^{\prime}, J^{\prime}\right)$ étant kählérienne, la dérivation covariante $D^{\prime}$ commute à $J^{\prime}$. Donc

$$
\frac{d E^{\prime}}{d s}-\frac{d E^{\prime \prime}}{d s}=\int_{M}\left[\left(J^{\prime} \frac{D^{\prime}}{\partial s} \lambda_{*} X_{j}, \lambda_{*} J X_{j}\right)+\left(J^{\prime} \lambda_{*} X_{j}, \frac{D^{\prime}}{\partial s} \lambda_{*} J X_{j}\right)\right]
$$

soit

$$
\begin{aligned}
\frac{d E^{\prime}}{d s}-\frac{d E^{\prime \prime}}{d s}=\int_{M}\left[-\left(\frac{D^{\prime}}{\partial s} \lambda_{*} X_{j}, J^{\prime} \lambda_{*} J X_{j}\right)+\left(J^{\prime} \lambda_{*} X_{j}, \frac{D^{\prime}}{\partial s} \lambda_{*} J X_{j}\right)\right] \\
=\int_{M}\left[-\left(D_{X_{j}}^{\prime} \frac{\partial \lambda}{\partial s}, J^{\prime} \lambda_{*} J X_{j}\right)+\left(J^{\prime} \lambda_{*} X_{j}, D_{J X}^{\prime} \frac{\partial \lambda}{\partial s}\right)\right] \\
=\int_{M}\left[-X_{j} \cdot\left(\frac{\partial \lambda}{\partial s}, J^{\prime} \lambda_{*} J X_{j}\right)+\left(\frac{\partial \lambda}{\partial s}, D_{X_{j}}^{\prime} J^{\prime} \lambda_{*} J X_{j}\right)\right. \\
\left.+J X_{j} \cdot\left(J^{\prime} \lambda_{*} X_{j}, \frac{\partial \lambda}{\partial s}\right)-\left(D_{J X_{j}}^{\prime} J^{\prime} \lambda_{*} X_{j}, \frac{\partial \lambda}{\partial s}\right)\right] .
\end{aligned}
$$

Définissons une 1-forme réelle $\omega_{s}$ sur $M$ en posant $\omega_{s}(X)=\left(\partial \lambda / \partial s, J^{\prime} \lambda_{*} J X\right)$. On peut alors écrire

$$
\begin{aligned}
-X_{j} \cdot\left(\partial \lambda / \partial s, J^{\prime} \lambda_{*} J X_{j}\right)+J X_{j} \cdot\left(\partial \lambda / \partial s, J^{\prime} \lambda_{*} X_{j}\right) \\
\quad=-X_{j} \cdot\left(\omega_{s}^{\sharp}, X_{j}\right)-J X_{j} \cdot\left(\omega_{s}^{\sharp}, J X_{j}\right) \\
\quad=-\left(D_{X_{j}} \omega_{s}^{\sharp}, X_{j}\right)-\left(D_{J X_{j}} \omega_{s}^{\sharp}, J X_{j}\right)-\left(\omega_{s}^{\sharp}, D_{X_{j}} X_{j}\right)-\left(\omega_{s}^{\sharp}, D_{J X_{j}} J X_{j}\right) .
\end{aligned}
$$

Comme les $\left\{X_{j}, J X_{j}\right\}$ forment une base orthonormée réelle de $(M, g)$, les deux premiers termes valent $\delta \omega_{s}$, et disparaissent par intégration. Il reste

$$
\begin{aligned}
\frac{d E^{\prime}}{d s}-\frac{d E^{\prime \prime}}{d s}= & \int_{M}\left[-\left(\frac{\partial \lambda}{\partial s}, J^{\prime} \lambda_{*} D_{X_{j}} X_{j}\right)+\left(\frac{\partial \lambda}{\partial s}, J^{\prime} \lambda_{*} D_{J X_{j}} X_{j}\right)\right] \\
& +\int_{M}\left[\left(\frac{\partial \lambda}{\partial s}, J^{\prime} D_{X_{j}}^{\prime} \lambda_{*} J X_{j}\right)-\left(\frac{\partial \lambda}{\partial s}, J^{\prime} D_{J X_{j}}^{\prime} \lambda_{*} X_{j}\right)\right] .
\end{aligned}
$$

(On a écrit deux intégrales séparees par abus de notation, tout devant entrer dans un seul signe somme.) Il vient 


$$
\begin{aligned}
\frac{d E^{\prime}}{d s}-\frac{d E^{\prime \prime}}{d s}= & \int_{M}-\left(\frac{\partial \lambda}{\partial s}, J^{\prime} \lambda_{*}\left(D_{X_{j}} J X_{j}-D_{J X_{j}} X_{j}\right)\right) \\
& +\int_{M}\left(\frac{\partial \lambda}{\partial s}, J^{\prime}\left(D_{X_{j}}^{\prime} \lambda_{*} J X_{j}-D_{J X_{j}}^{\prime} \lambda_{*} X_{j}\right)\right) \\
= & \int_{M}\left[-\left(\frac{\partial \lambda}{\partial s}, J^{\prime} \lambda_{*}\left[X_{j}, J X_{j}\right]\right)+\left(\frac{\partial \lambda}{\partial s}, J^{\prime} \lambda_{*}\left[X_{j}, J X_{j}\right]\right)\right]=0 .
\end{aligned}
$$

De ce résultat et de la relation $E^{\prime}+E^{\prime \prime}=E$, on tire

$$
d E^{\prime} / d s=d E^{\prime \prime} / d s=\frac{1}{2} d E / d s .
$$

On en déduit immédiatement le lemme:

Lemme. a) Les fonctionnelles $E, E^{\prime}, E^{\prime \prime}$ sur $\mathscr{C}^{\circ}\left(M, M^{\prime}\right)$ ont les mêmes points critiques.

b) Si le long d'un chemin de $\mathscr{C}^{\infty}\left(M, M^{\prime}\right)$, l'une de ces fonctionnelles est constante, les deux autres le sont aussi.

Voyons maintenant comment s'exprime la formule de la variation seconde dans des bases orthonormées complexes de $(M, g, J),\left\{X_{j}\right\}_{j=1}^{n}$. Notons que les $X_{j}$ réunis aux $J X_{j}$ forment des bases orthonormées réelles de $(M, g)$. On a donc d'après la formule (7):

$$
\begin{aligned}
\frac{\partial^{2} E}{\partial s \partial t}= & -\int_{M}\left(\frac{D^{\prime}}{\partial s} \frac{\partial \lambda}{\partial t}, \tau\left(\lambda_{s t}\right)\right) \\
& +\int_{M}\left[\sum_{j=1}^{n}\left(D_{X_{j}}^{\prime} \frac{\partial \lambda}{\partial s}, D_{X_{j}}^{\prime} \frac{\partial \lambda}{\partial t}\right)+\sum_{j=1}^{n}\left(D_{J X_{j}}^{\prime} \frac{\partial \lambda}{\partial s}, D_{J X_{j}}^{\prime} \frac{\partial \lambda}{\partial t}\right)\right] \\
- & -\int_{M}\left[\sum_{j=1}^{n} R^{\prime}\left(\lambda_{*} X_{j}, \frac{\partial \lambda}{\partial s}, \lambda_{*} X_{j}, \frac{\partial \lambda}{\partial t}\right)\right. \\
& \left.+\sum_{j=1}^{n} R^{\prime}\left(\lambda_{*} J X_{j}, \frac{\partial \lambda}{\partial s}, \lambda_{*} J X_{j}, \frac{\partial \lambda}{\partial t}\right)\right] .
\end{aligned}
$$

Nous allons transformer le dernier terme en nous plaçant dans les complexifiés des divers fibrés envisagés. Considérons d'abord les parties de type $(1,0)$ et $(0,1) \operatorname{des} X_{j}$ :

$$
\xi_{j}=\frac{1}{\sqrt{2}}\left(X_{j}-i J X_{j}\right), \quad \bar{\xi}_{j}=\frac{1}{\sqrt{2}}\left(X_{j}+i J X_{j}\right),
$$

d'où

$$
X_{j}=\frac{1}{\sqrt{2}}\left(\xi_{j}+\bar{\xi}_{j}\right), \quad J X_{j}=\frac{i}{\sqrt{2}}\left(\xi_{j}-\bar{\xi}_{j}\right)
$$


Posant $U=\sum_{j=1}^{n} R^{\prime}\left(\lambda_{*} X_{j}, \frac{\partial \lambda}{\partial s}, \lambda_{*} X_{j}, \frac{\partial \lambda}{\partial t}\right)+\sum_{j=1}^{n} R^{\prime}\left(\lambda_{*} J X_{j}, \frac{\partial \lambda}{\partial s}, \lambda_{*} J X_{j}, \frac{\partial \lambda}{\partial t}\right)$, un calcul immédiat montre que

$$
U=\sum_{j=2}^{n} 2 R^{\prime}\left(\lambda_{*} \xi_{j}, \frac{\partial \lambda}{\partial s}, \lambda_{*} \bar{\xi}_{j}, \frac{\partial \lambda}{\partial t}\right) .
$$

Définissons une forme biquadratique sur $T_{y} M^{\prime}, y \in M^{\prime}$, par $\alpha(u, v)=R^{\prime}(u, \bar{v}, \bar{u}, v)$. On a $\overline{\alpha(u, v)}=R^{\prime}(\bar{u}, v, u, \bar{v})=R^{\prime}(u, \bar{v}, \bar{u}, v)=\alpha(u, v)$. Donc $\alpha$ est réelle. De plus $\alpha(\lambda u, v)=\alpha(u, \lambda v)=|\lambda|^{2} \alpha(u, v)$ pour tout $\lambda \in C$. Donc $\alpha$ est bihermitienne.

On dit que $\alpha$ est la courbure kählérienne complète de $\left(M^{\prime}, J^{\prime}, g^{\prime}\right)$. On a alors:

$$
U=2 \sum_{j=1}^{n} \alpha\left(\lambda_{*} \xi_{j}, \frac{\partial \lambda}{\partial s}\right)
$$

On a alors un résultat partiellement analogue à la proposition 2:

Proposition 3. Soit $f:(M, g, J) \rightarrow\left(M^{\prime}, g^{\prime}, J^{\prime}\right)$ une application holomorphe.

a) $S i\left(M^{\prime}, J^{\prime}, g^{\prime}\right)$ est à courbure kählérienne complète non positive sur $f(M)$, l'opérateur $L$ est semi défini positif et son noyau est formé de champs parallèles le long de $f$.

b) Si $\left(M^{\prime}, J^{\prime}, g^{\prime}\right)$ est à courbure kählérienne complète non positive au voisinage de $f(M), f$ est LSIC au sens $C^{0}$. Il existe même dans $\mathscr{C}^{\infty}\left(M, M^{\prime}\right)$ un voisinage $\mathfrak{A}$ de $f$ au sens $C^{0}$ tel que l'on ait $E(h) \geq E(f)$ pour toute $h \in \mathfrak{A}$, l'égalité étant atteinte si et seulement si h est holomorphe, auquel cas il existe un champ $u \in \Gamma\left(f^{*} T M^{\prime}\right)$, parallèle le long de $f$, tel que l'on ait $h=\exp _{f}^{\prime} u$; et de plus les applications intermédiaires $\exp _{f}^{\prime} s u, s \in[0,1]$, sont holomorphes.

La démonstration est la même que pour la proposition 2 . Il suffit de remarquer en plus que si $E$ est constante le long de l'homotopie $\exp _{f}^{\prime} s u$, il en est de même de $E^{\prime \prime}$, donc que $E^{\prime \prime}$ reste nulle le long de cette homotopie.

\section{Cas où $f$ est l'identite}

Soit $(M, g)$ une variété riemannienne connexe compacte. L'application identique de $(M, g)$ dans elle-même est harmonique. Cherchons ce qu'est ici l'opérateur $L$. Des bases orthonormées locales $\left\{X_{i}\right\}_{i=1}^{n}$ étant choisies, on a (formule $\left(6^{\prime}\right)$ )

$$
L u=\sum_{i=1}^{n} D_{D_{X_{i}} X_{i}} u-\sum_{i=1}^{n} D_{X_{i}} D_{X_{i}} u-\rho(u),
$$

où $\rho$ est l'opérateur autoadjoint ponctuel associé au tenseur de Ricci. La forme bilinéaire correspondante ${ }^{1}$ sera aussi notée $\rho$. On a alors

${ }^{1}$ Dans ce qui suit, nous identifierons les divers espaces de tenseurs de même ordre mais de types différents, au moyen des isomorphismes canoniques entre ces divers espaces résultant de la structure riemannienne. Comme celle-ci est fixée, il n'y a pas d'ambiguité, et nous ne mentionnerons pas explicitement ces isomorphismes. 


$$
L u=\sum_{i=1}^{n} \underbrace{}_{i} \otimes D X_{i} \otimes D u-\sum_{i=1}^{n} \underbrace{}_{i} \otimes D\left(X^{i} \otimes D u\right)-\rho(u),
$$

où le symbole $\otimes$ désigne le produit tensoriel contracté. Donc

$$
L u=-\sum_{i=1}^{n} X_{i} \otimes X_{i} \otimes D^{2} u-\rho(u)
$$

En coordonnées locales,

$$
(L u)^{i}=-D^{j} D_{j} u^{i}-\rho_{j}^{i} u^{j}=\Delta u^{i}-\rho_{j}^{i} u^{j} .
$$

D'après $\left[7\right.$, formule 1.6], on a $\Delta u^{i}=(\Delta u)^{i}-\rho_{j}^{i} u^{j}$, d'où

$$
(L u)^{i}=(\Delta u)^{i}-2 \rho_{j}^{i} u^{j} .
$$

Finalement

$$
L u=\Delta u-2 \rho(u) .
$$

D'autre part, d'après [7, formule 2.1] on a

$$
\langle u, \Delta u\rangle=2\|D u\|^{2}+\int_{M} \rho(u, u),
$$

d' où

$$
\langle u, L u\rangle=2\|D u\|^{2}-\int_{M} \rho(u, u)
$$

On obtient alors une forme un peu différente de la proposition 2.

Proposition 4. a) Si $(M, g)$ est à courbure de Ricci non positive, l'opérateur $L$ est semi-défini positif et son noyau est l'espace des champs parallèles sur $(M, g)$. En particulier les isométries infinitésimales de $(M, g)$ sont parallèles.

b) Si $(M, g)$ est à courbure de Ricci définite négative, l'identité est LSIC au sens de la topologie $C^{1}$. Il existe même un voisinage $\mathfrak{X}$ de $\mathrm{Id}$ au sens $C^{1}$ tel que l'on ait $E(h) \geq E(\mathrm{Id})$ pour tout $h \in \mathfrak{A}$, l'égalité étant atteinte si et seulement si $h=$ Id.

a) découle trivialement de la formule (9). Pour b), soit $K$ l'ensemble des $\left(x_{1}, \cdots, x_{n}\right) \in(T M)^{n}$ qui forment une base orthonormée. Choisissons dans $(T M)^{n}$ un voisinage $\Omega$ de $K$ sur lequel on a

$$
\sum_{i=1}^{n} R\left(x_{i}, v, x_{i} v\right) \leq 0 \quad \text { pour tout }\left(x_{1}, \cdots, x_{n}\right) \in \Omega \text { et tout } v \in T M .
$$

On raisonnera comme à la proposition 2 en prenant $\mathfrak{U}$ assez petit pour que 
les $\left(\lambda_{s^{*}}\left(X_{1}\right), \cdots, \lambda_{s^{*}}\left(X_{n}\right)\right)$ soient dans $\Omega$. On aura alors pour tout $h \in \mathfrak{A}$, $E(h) \geq E($ Id), l'égalité étant atteinte si et seulement si $h=\exp u$ pour un certain champ parallèle $u$. Mais si $(M, g)$ est à courbure de Ricci définie négative, il n'y a pas de champ parallèle non nul, donc $h=\mathrm{Id}$.

Remaque 4.1. Une variété à courbure de Ricci non positive telle que l'identité ne soit pas LSIC au sens $C^{0}$ doit etre bien étrange.

Remarque 4.2. Si la courbure de Ricci est définie négative en un point au moins, il ne peut pas exister de champs parallèles non nuls et toute isométrie infinitésimale est nulle. On retrouve le résultat suivant, dû à Bochner [3]:

Soit Aut $(M, g)$ le groupe des isométries d'une variété riemannienne $(M, g)$.

Si $(M, g)$ est compacte et à courbure de Ricci non positive, il existe un tore $T$ et un groupe fini $F$ tels que l'on ait une suite exacte

$$
0 \rightarrow T \rightarrow \text { Aut }(M, g) \rightarrow F \rightarrow 0 .
$$

Si de plus la courbure de Ricci est définie négative en un point, Aut $(M, g)$ est fini.

Nous pouvons préciser ce résultat par la propriété de rigidité suivante:

Proposition 5. Soient $g$ et $g^{\prime}$ deux métriques à courbure sectionnelle non positive sur la variété différentiable connexe compacte $M$. Dans les suites exactes de Bochner $0 \rightarrow T \rightarrow$ Aut $(M, g) \rightarrow F \rightarrow 0$ et $0 \rightarrow T^{\prime} \rightarrow$ Aut $\left(M, g^{\prime}\right) \rightarrow F^{\prime} \rightarrow 0$, les tores $T$ et $T^{\prime}$ ont même dimension. Ou encore les espaces $P$ et $P^{\prime}$ de champs parallèles sur $(M, g)$ et $\left(M^{\prime}, g^{\prime}\right)$, algèbres de Lie de $T$ et $T^{\prime}$ ont même dimension.

Soit $\mathscr{C}_{0}^{\infty}(M)$ l'espace des applications $C^{\infty}$ de $M$ dans $M$ homotopes à l'identité. Soit $H_{0}(M, g)$ l'espace des applications harmoniques de $M$ dans $M$ homotopes à l'identité. D'après la théorie de Eells-Sampson-Hartmann, $\mathscr{C}_{0}^{\infty}(M)$ et $H_{0}(M, g)$ ont même type d'homotopie. Par ailleurs d'après Hartmann, si $h \in H_{0}(M, g)$ il existe une homotopie $\lambda$ de Id à $h$ telle que pour tout $x \in M$, l'application $\lambda_{x}: s \rightarrow \lambda(x, s)$ est alors constante, de sorte que le champ $u=$ $(\partial \lambda / \partial s)_{s=0}$ est parallèle. Alors $h=\exp u$ est dans $T$. Donc $H_{0}(M, g)=T$. Ainsi $T$ et $T^{\prime}$ ont même type d'homotopie, donc même dimension.

Remarque 5.1. Supposons même que $(M, g)$ et $\left(M^{\prime}, g^{\prime}\right)$ soient deux variétés riemanniennes connexes compactes à courbure sectionnelle non positive, $M$ et $M^{\prime}$ ayant même type d'homotopie. Dans ce cas $M$ et $M^{\prime}$ ont même type d'homotopie différentiable, en ce sens qu'il existe des applications $C^{\infty}, \varphi$ : $M \rightarrow M^{\prime}$ et $\Psi: M^{\prime} \rightarrow M$ telles que $\Psi \circ \varphi$ et $\varphi \circ \Psi$ soient différentiablement homotopes a $\operatorname{Id}_{M}$ et $\operatorname{Id}_{M}$, respectivement. Par suite $\mathscr{C}_{0}^{\infty}(M)$ et $\mathscr{C}_{0}^{\infty}\left(M^{\prime}\right)$ ont même type d'homotopie, donc $\operatorname{dim} T=\operatorname{dim} T^{\prime}$. La dimension du plus grand groupe connexe d'isométries est donc, pour les variétés riemanniennes considérées, un invariant du type d'homotopie. Par des méthodes tout à fait différentes, Lawson et Yau [6] ont encore précisé ce résultat, en montrant que cet invariant est égal au rang du centre du groupe fondamental.

Donnons maintenant une caractérisation variationnelle des isométries infinitésimales. 
Proposition 6. Soit u un champ de vecteurs sur $(M, g)$. Les trois propriétés suivantes sont équivalentes:

i) $u$ est une isométrie infinitésimale.

ii) $u$ satisfait les équations $L u=o, \delta u=o$.

iii) $u$ rend la variation du volume stationnaire au premier ordre et la variation de l'énergie stationnaire au second ordre au voisinage de l'identité.

Rappelons d'abord que si $\mathscr{X}(M)$ désigne l'espace des champs de vecteurs sur $M$, et $\mathscr{S}^{2}(M)$ l'espace des 2-formes différentielles symétriques sur $M$ muni du produit scalaire défini dans l'introduction, on a les opérateurs $\delta^{\prime}: \mathscr{S}^{2}(M)$ $\rightarrow \mathscr{X}(M)$ et $\delta^{\prime *}: \mathscr{X}(M) \rightarrow \mathscr{S}^{2}(M)$, adjoints l'un de l'autre, définis par

$$
\begin{gathered}
\left(\delta^{\prime} h\right)_{i}=-D^{j} h_{j i}, \\
\left(\delta^{*} u\right)_{i j}=D_{i} u_{j}+D_{j} u_{i} .
\end{gathered}
$$

Rappelons qu'on a

$$
\delta^{*} u=\mathscr{L}_{u} g
$$

où $\mathscr{L}_{u}$ désigne la dérivée de Lie relative au champ $u$. Alors les isométries infinitésimales sont exactement les éléments du noyau de $\delta^{*}$. On a

$$
\begin{aligned}
\left(\delta^{\prime} \delta^{\prime *} u\right)^{i} & =-D^{j}\left(D_{j} u^{i}+D^{i} u_{j}\right)=\Delta u^{i}-D^{j} D^{i} u_{j} \\
& =(\Delta u)^{i}-\rho_{j}^{i} u^{j}-D^{i} D^{j} u_{j}-\rho_{j}^{i} u^{j} \\
& =(L u)^{i}+D^{i} \delta u,
\end{aligned}
$$

d' où

$$
\begin{gathered}
\delta^{\prime} \delta^{*} u=L u+d \delta u \\
\left\langle\delta^{\prime *} u, \delta^{*} u\right\rangle=\langle u, L u\rangle+\langle\delta u, \delta u\rangle .
\end{gathered}
$$

i) $\Rightarrow$ ii) Si $u$ est une isométrie infinitésimale, $\delta u=0$ et $\delta^{\prime *} u=0$ d'où par la formule (13), $L u=0$.

ii) $\Rightarrow$ iii) Il est classique que $\delta u=0$ équivaut à dire que $u$ rend la variation du volume stationnaire au premier ordre. D'autre part, si $L u=0,\langle u, L u\rangle$ $=0$, ce qui équivaut à dire que $u$ rend la variation de l'énergie stationnaire au second ordre.

iii) $\Rightarrow$ i) L'hypothèse équivaut à $\delta u=0$ et $\langle u, L u\rangle=0$, d'où par la formule $\left(13^{\prime}\right),\left\langle\delta^{\prime *} u, \delta^{*} u\right\rangle=0$, d'où $\delta^{\prime}{ }_{*} u=0$, et $u$ est une isométrie infinitésimale.

Remarque 6.1. L'équivalence i) $\Longleftrightarrow$ ii) a été prouvée par Lichnérowicz en [7, p. 129].

Considérons le cas d'une variété kählérienne compacte $(M, g, J)$. Pour de telles variétés on a un résultat analogue au théorème de Bochner: Si la première 
classe de Chern $C_{1}(M)$ est non positive pour la structure complexe $J$, le plus grand groupe connexe de transformations holomorphes de $(M, J)$ est un tore complexe. Son algèbre de Lie s'identifie à celle des champs de vecteurs complexes de type $(1,0)$ holomorphes. Voir $[8$, théorème 1$]$.

Nous obtenons un résultat de rigidité analogue à la proposition 4.

Proposition 7. Soient $(g, J)$ et $\left(g^{\prime}, J^{\prime}\right)$ deux structure kählériennes à courbure sectionelle non positive sur la variété différentiable connexe compacte $M$. Si $T$ et $T^{\prime}$ sont les plus grands groupes connexes de transformations holomorphes de $(M, J)$ et $\left(M, J^{\prime}\right)$, alors $T$ et $T^{\prime}$ ont même dimension. Ou encore, si $\mathscr{H}$ et $\mathscr{H}^{\prime}$ sont les espaces de champs de vecteurs complexes de type $(1,0)$ holomorphes sur $(M, J)$ et $\left(M, J^{\prime}\right)$, $\mathscr{H}$ et $\mathscr{H}^{\prime}$ ont même dimension.

Le raisonnement est le même qu'à la proposition 5 , mis à part qu'ici il s'agit de montrer que toute application harmonique $h$ homotope à l'identité est une transformation holomorphe. Choisissant une homotopie d'Hartman de la forme $\exp s u, s \in[0,1]$, on voit d'abord que $h$ est inversible. D'autre part $E^{\prime \prime}$ est constamment nulle le long de l'homotopie, donc $h$ est holomorphe.

Pour voir un exemple où $L$ n'est pas semi défini positif, étudions le cas des sphères.

Proposition 8. a) Sur $\left(S^{2}, g_{0}\right)$, L est semi défini positif et son noyau est de dimension 3.

b) $\operatorname{Sur}\left(S^{n}, g_{0}\right), n \geq 3, L$ admet l'unique valeur propre négative $2-n$, avec la multiplicité $n+1$. Les autres valeurs propres sont strictement positives.

On a $L=\Delta-2(n-1)$ Id de sorte que les vecteurs propres de $L$ sont aussi ceux de $\Delta$. D'après $[7$, p. 134], ces vecteurs se partagent en fermés et cofermés, et pour les cofermés on a une minoration des valeurs propres correspondantes:

$$
\lambda \geq 2(n-1)
$$

d'où $\lambda-2(n-1) \geq o$. $L$ est semi défini positif sur les vecteurs cofermés, ce qui est d'ailleurs absolument général d'après la formule $\left(13^{\prime}\right)$. Considérons maintenant un vecteur fermé. Les sphères étant simplement connexes il s'écrit $d f$ où $f$ est une certaine fonction que l'on peut supposer d'intégrale nulle.

On a $\Delta d f=\lambda d f$, soit encore $d(\Delta f-\lambda f)=0$ car $d$ et $\Delta$ commutent. Donc $\Delta f-\lambda f$ est une constante, qui est nulle d'après le choix de $f$. On en conclut que les vecteurs propres de $L$ susceptibles de correspondre à des valeurs propres négatives sont les gradients des fonctions propres du laplacien.

Le spectre du laplacien sur les fonctions est calculé pour les sphères dans [2, proposition C.I. 1 du chapitre III]. La première valeur propre est $n$ avec la multiplicité $n+1$. La première valeur propre de $L$ correspondant à des vecteurs fermés est donc $2-n$ avec la multiplicité $n+1$. Pour $n=2$ on trouve bien que $L$ est semi défini positif et que son noyau est de dimension 3 . Pour $n \geq 3$, on trouve la valeur propre négative $2-n$, avec la multiplicité 
$n+1$. La valeur propre suivante est $2(n+1)-2(n-1)=4$.

Remarque 8.1. Toujours d'après [2], les vecteurs propres relatifs à la valeur propre négative de $L$ sont les gradients des fonctions $f$ définies comme suit: $\left(S^{n}, g_{0}\right)$ étant plongée dans $\left(\boldsymbol{R}^{n+1}, g_{0}\right)$, choisissons un vecteur $z \in \boldsymbol{R}^{n+1}$. Alors $f_{z}(x)=g_{0}(x, z)$.

Si $u$ est un tel vecteur propre, on peut même montrer que l'énergie du diffémorphisme exp tu tend vers 0 quand $t$ tend vers l'infini (cf. [4, p. 130]).

\section{Bibliographie}

[1] M. Berger, On geodesics in Riemannian geometry, Tata Institute, Bombay, 1965.

[2] M. Berger, P. Gauduchon \& E. Mazet, Le spectre d'une variété riemannienne, Lecture Notes in Math. Vol. 194, Springer, Berlin, 1971.

[3] S. Bochner, Vector fields and Ricci curvature, Bull. Amer. Math. Soc. 52 (1946) 776-797.

[ 4 ] J. Eells Jr. \& J. H. Sampson, Harmonic mappings of riemannian mannifolds, Amer. J. Math. 86 (1964) 109-160.

[ 5 ] P. Hartman, On homotopic harmonic maps, Canad. J. Math. 19 (1967) 673-687.

[6] H. B. Lawson, Jr. \& S. T. Yau, Compact manifolds of nonpositive curvature, J. Differential Geometry 7 (1972) 211-228.

[ 7 ] A. Lichnerowicz, Géométrie des groupes de transformations, Dunod, Paris, 1958.

] 8 [ - Variétés kählériennes et première classe de Chern, J. Differential Geometry 1 (1967) 195-223.

[9] - Applications harmoniques et variétés kählériennes, Rend. Sem. Mat. Fis. Milano 39 (1969) 1-12.

Ecole Polytechnique, Paris 